\title{
Multidisciplinary, multimodal approach for a child with a traumatic facial scar
}

\author{
Shehla Admani, MD, ${ }^{1}$ Jeffrey W Gertner, MD, ${ }^{2}$ Amanda Gosman, MD, ${ }^{1,3}$ Peter R Shumaker, MD, ${ }^{2}$ \\ Nathan S Uebelhoer, DO, ${ }^{2,4^{*}}$ and Andrew C Krakowski, MD ${ }^{1,3}$
}

\section{Abstract}

The treatment of disfiguring and disabling scars remains a field of active study, reinvigorated with recent advances in techniques and technologies. A variety of approaches can be utilized depending on scar characteristics, location, degree of tissue loss, and associated contractures. Just as traumatic scars can be complex and heterogeneous, the corresponding paradigm for treatment must also be flexible and multimodal for optimal improvement. This report describes a 3-year-old girl with a "mixed" (atrophic/hypertrophic), violaceous, contracted facial scar from a dog bite. It was treated with a novel approach utilizing a multidisciplinary pediatric scar team to combine autologous fat grafting, ablative fractional laser resurfacing, pulsed-dye laser, and laser-assisted delivery of a corticosteroid as concurrent, multimodal therapy to optimize the outcome.

Semin Cutan Med Surg 34:24-27 (c) 2015 Frontline Medical Communications

$\longrightarrow$ hildren are particularly prone to trauma due to their inquisitive nature and relative lack of experience and judgment. Most of the time, this trauma is minor and heals with inconsequential scarring. Unfortunately, children sometimes do experience severe trauma resulting in disfiguring and disabling scars. There are a multitude of approaches that can be utilized to address symptomatic scars that are selected based upon individual patient characteristics (eg, increased anxiety, posttraumatic stress disorder, decreased social interaction) and specific scar characteristics (eg, conspicuous or not, anatomic location, size, thickness, depth of injury, texture, color, attachment to underlying tissues, presence of contractures), among other considerations. A single provider from a single specialty may not possess the breadth of experience to adequately address all of these factors; as a result, it may be beneficial to involve a multidisciplinary team in the coordinated management of such patients.

This approach is not new, and there are many reports in the literature of multidisciplinary teams used in the management of vas-

\footnotetext{
${ }^{1}$ University of California, San Diego, La Jolla, California.

${ }^{2}$ Naval Medical Center, San Diego, California.

${ }^{3}$ Rady Children's Hospital, San Diego, California.

${ }^{4}$ The Aroostook Medical Center, Presque Isle, Maine.

*Dr Uebelhoer is now with The Aroostook Medical Center.

Disclosures: Work was performed at Rady Children's Hospital, San Diego

with the Department of Pediatrics, Division of Pediatric and Adolescent Dermatology. No external funding was secured for this study. The authors have no financial relationships relevant to this article to disclose.

Correspondence: Andrew C. Krakowski, MD; Director, Kids' Scar Treatment and Revision (S.T.A.R.) Program; Rady Children's Hospital, San Diego; 8010 Frost Street, Suite 602; San Diego CA, 92123. E-mail: akrakowski@rchsd.org
}

cular lesions, cleft lip and palate, and pediatric burns. ${ }^{1-3}$ Herein, we report a multidisciplinary approach to traumatic scars with the combination of autologous fat transfer (AFT), a series of ablative fractional laser resurfacing (AFR) and pulsed-dye laser (PDL) treatments, and laser-assisted delivery of corticosteroids to address a challenging traumatic scar located conspicuously on the face of a young girl.

\section{Case report}

A 3-year-old girl initially presented with a history of a $7 \mathrm{~cm} \mathrm{x} 2 \mathrm{~cm}$ scar resulting from a dog bite to the right cheek inflicted 21 months earlier. Initial reconstruction at an outside hospital involved a tissue expander to help provide additional tissue to cover the defect. The resulting "mixed" scar consisted of areas of atrophy and hypertrophy, prominent erythema, and marked asymmetry secondary to focal loss of subcutaneous tissue. Significant skin scar contractures and asymmetry were noted when the child smiled (Figures 1 and 2). The patient also appeared to display an inordinate level of anxiety regarding the presence of her scar and her perception of it by others with whom she interacted.

The child's pediatrician referred her to a regional pediatric scar clinic for coordinated care with available expertise in plastic surgery, pediatric and procedural dermatology, trauma counseling, and physical and occupational therapy, among other specialties. In direct consultation with the patient and her family, a multimodal treatment course was recommended including AFT to help mitigate volume loss, a series of AFR and PDL treatments to improve scar texture, pliability, mobility, and erythema, and corticosteroids for areas of hypertrophy. Formal counseling was recommended to help the patient overcome any residual posttraumatic stress associated with the dog bite incident itself.

After obtaining informed consent, the patient was placed under general anesthesia due to her age and the extent and location of her injuries. The entire scar sheet was then treated using an ablative microfractionated 10600-nm carbon dioxide $\left(\mathrm{CO}_{2}\right)$ laser (Ultrapulse Encore Deep FX, Lumenis, Ltd, Yokneam, Israel) at a pulse energy of $15 \mathrm{~mJ}$ and a density of $15 \%$. Immediately after the ablative fractional laser treatment, her plastic surgery team performed AFT to the scarred area. Fat was harvested through an entry portal just inferior to the umbilicus, and after processing through a filtration system (Puregraft LLC, Solana Beach, California, USA), approximately $5 \mathrm{cc}$ of fat was available for transfer. The entire quantity was injected into the right cheek deep into the AFR-treated scar via a small incision near the right oral commissure. The patient tolerated both procedures well and healed without complications.

The patient ultimately received a total of nine combination laser treatments, including AFR and PDL, at a minimum of 8-week intervals over the next 19 months. In all the laser sessions, the scars were first treated with the PDL since mild focal erythema, swell- 

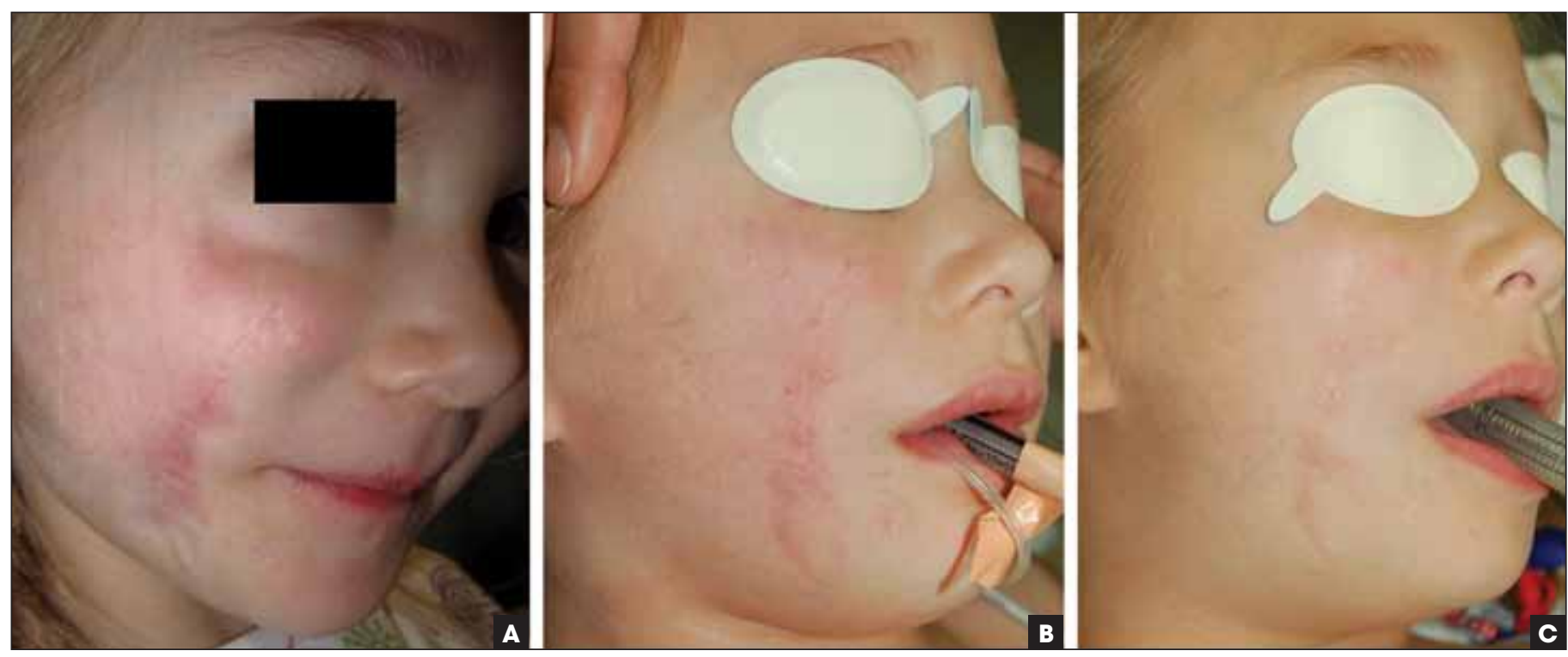

FIGURE 1. Scar evolution over 19 months. A) shows a 3-year-old girl who presented with a "mixed" (ie, atrophic and hypertrophic) scar resulting from a dog bite to the right cheek inflicted 21 months earlier. Marked erythema, irregular texture, and skin scar contractures are noted. B) shows the same patient 10 months after her initial combined AFR and AFT surgery, immediately prior to her 5th combined AFR and PDL treatment. Improvements in erythema and scar texture are evident. C) shows the same patient 19 months after her initial presentation, immediately prior to her 9th combined AFR and PDL treatment. Scar texture, erythema, and skin scar contractures are greatly improved.

ing, and scant serous discharge generally follows AFR treatments within several minutes. Erythematous portions of the scar were initially treated with a 595-nm PDL (Vbeam; Candela Corporation, Wayland, Massachusetts, USA) with a $7 \mathrm{~mm}$ spot size, fluence of $8 \mathrm{~J} / \mathrm{cm}^{2}$, and $1.5 \mathrm{~ms}$ pulse width. Serial PDL treatments were repeated at identical spot-size and pulse-width settings, with a range of fluence settings of 8 to $11 \mathrm{~J} / \mathrm{cm}^{2}$ to reach a clinical endpoint of minimal purpura. Subsequent AFR pulse energies ranged from $15 \mathrm{~mJ}$ to $25 \mathrm{~mJ}$ depending on estimated scar thickness in a particular area, with corresponding treatment densities of $10 \%-5 \%$ with a single pass in a stamped pattern. Treatment densities were decreased with increasing pulse energy to minimize cumulative thermal injury.

In four of her treatment sessions, the patient received adjunctive laser-assisted delivery of triamcinolone acetonide $(40 \mathrm{mg} / \mathrm{mL})$ to the areas of greatest scar hypertrophy. In this technique, intralesional-grade triamcinolone acetonide suspension is "dripped" over the treatment site and rubbed gently over the ablated columns within approximately two minutes after laser treatment to facilitate dermal delivery through capillary action. ${ }^{4}$ Immediately after all laser treatments, a petrolatum-based ointment was applied (Aquaphor ointment, Beiersdorf, Inc, Wilton, Connecticut, USA) and continued 2-3 times a day until complete re-epithelialization occurred (generally 2-3 days). No postoperative complications other than laser-induced purpura were reported and the patient was able to resume essentially normal activity one day after her laser surgeries.

At the completion of her 9th treatment, the patient was noted to have markedly improved scar texture, scar color, and facial symmetry. Erythema, while persistent in the most atrophic areas of the patient's scar, was significantly reduced from initial presentation. The patient had overcome any residual posttraumatic stress and, ultimately, accepted a dog as a household pet. Her anxiety with the scar itself and her perceived reaction by others to it had nearly resolved. She will continue to see the pediatric dermatologist for laser treatment of her persistent erythema.

\section{Discussion}

A variety of treatment modalities have been used to improve the symptoms and appearance of scars in both adults and pediatric patients, including intralesional triamcinolone acetonide, ablative and nonablative fractional laser resurfacing, PDL, cryotherapy, and silicone-based products. ${ }^{5}$ AFT is an emerging option in adults for aesthetic facial recontouring, the treatment of human immunodeficiency virus associated lipodystrophy, and for various scar types. ${ }^{6-9}$ Early indications are that the effects are more than simple volume replacement, with graft components mediating a dynamic remodeling process that remains to be fully elucidated. ${ }^{10,11}$

In the pediatric population, AFT has been reported for the treatment of facial malformations due to Goldenhar syndrome, Treacher Collins syndrome, and hemifacial microsomia. ${ }^{12}$ Large complex scars may require multiple interventions performed either concurrently or in a step-wise fashion for optimal results. ${ }^{13}$ A correlate in the pediatric population can be seen with the treatment of secondary cleft-lip deformity. AFT and composite grafting can be performed using skin and subcutaneous fat to address both volume loss and overlying scarring. ${ }^{14,15}$

Our patient received multiple modalities concurrently and in series including AFT, AFR, PDL, and laser-assisted corticosteroid delivery to address varying and overlapping aspects of the scar. Specifically, the associated volume loss was treated with AFT. The textural irregularity and scar contractures were addressed with serial AFR treatments and laser-assisted delivery of a corticosteroid. Lastly, PDL was employed to reduce the persistent erythema. Each of these modalities independently has varying degrees of support 

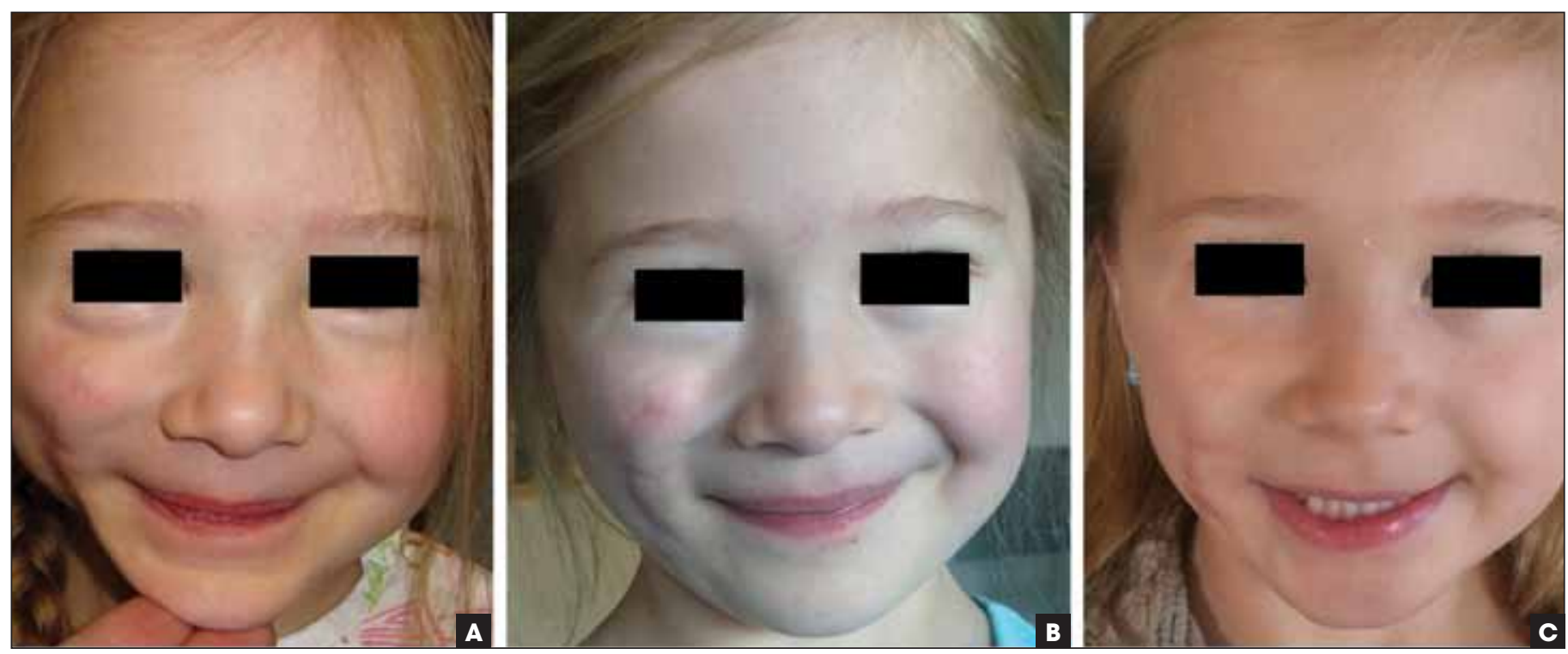

FIGURE 2. Sustained volume replacement. A) Patient at initial presentation to her multidisciplinary scar team. Marked right cheek volume loss and skin scar contractures are evident with smiling. B) Patient 8 months after her initial combined AFR and AFT surgery (photo provided by patient's mother). Right cheek volume is much improved as are the contractures. C) Patient 18 months after her initial AFR and AFT. Note the sustained volume improvement in the right cheek (photo provided by patient's mother).

in the literature for the treatment of scars, and each on their own are associated with the induction of scar remodeling. PDL and intralesional corticosteroids have had a role in scar treatment for decades. AFR is a very promising and relatively recent addition to the scar armamentarium, though few reports specifically address the use of AFR for scars in children. ${ }^{16}$ Krakowski et al recently reported efficacy for the functional enhancement of pediatric scar contractures. ${ }^{17}$ Combinations of AFT with ablative and nonablative fractional laser resurfacing and platelet-rich plasma have been reported to enhance scar treatment. ${ }^{18,19}$ However, to our knowledge, the combination of AFT and the concurrent adjunctive modalities of AFR, PDL, and laser-assisted delivery of a corticosteroid has not previously been reported for traumatic scar management in the pediatric population.

Given the single case report, lack of control, and multiple concurrent modalities employed, it is not possible to discern the exact individual contributions of the various modalities to the observed improvements and whether there was any synergy between them. While there may have been some spontaneous improvement in the patient's scar over time, the fact that the scar was 21 months old at treatment initiation suggests that spontaneous healing was not a major factor in the outcome of this patient. Clearly, larger controlled studies will be required to confirm and elucidate these observations. However, this coordinated, multidisciplinary, multimodal approach was felt by the authors to be more effective than any monotherapy.

There were both theoretical and practical considerations for the combination described above. Given the need for general anesthesia, there was a desire to minimize anesthetic events. Furthermore, it was felt that photomechanical fenestration of the restrictive scar with AFR immediately prior to the AFT would enhance tissue filling and fat distribution and might even augment the subsequent remodeling response. In the view of the authors, there may also be a theoretical advantage to AFR pretreatment in the months prior to AFT to enhance long-term scar pliability and quality prior to attempting volume correction. This young girl's case emphasizes the need to take into account individual scar characteristics in order to tailor appropriate treatments and optimize clinical outcomes. Likewise, it supports the notion that certain scars may benefit from multiple, coordinated treatment modalities, which may be performed concurrently to optimize scar rehabilitation.

Disclaimer: The views expressed in this article are those of the authors and do not necessarily reflect the official policy or position of the Department of the Navy, Department of Defense, or the United States Government. Drs Gertner and Shumaker are military service members. This work was prepared as part of their official duties. Title 17, USC, $\S 105$ provides that Copyright protection under this title is not available for any work of the United States Government. Title 17, USC, $\S 101$ defines a US Government work as a work prepared by a military service member or employee of the US Government as part of that persons official duties.

\section{References}

1. Mathes EF, Haggstrom AN, Dowd C, Hoffman WY, Frieden IJ. Clinical characteristics and management of vascular anomalies: findings of a multidisciplinary vascular anomalies clinic. Arch Dermatol. 2004;140(8):979-983.

2. Robin NH, Baty H, Franklin J, et al. The multidisciplinary evaluation and management of cleft lip and palate. South Med J. 2006;99(10):1111-1120.

3. Warden GD, Brinkerhoff C, Castellani D, Rieg LS. Multidisciplinary team approach to the pediatric burn patient. QRB Qual Rev Bull. 1988;14(7):219-226.

4. Waibel JS, Wulkin AJ, Shumaker PR. Treatment of hypertrophic scars using laser and laser assisted corticosteroid delivery. Lasers Surg Med. 2013;45(3):135-140.

5. Gold MH, Berman B, Clementoni MT, Gauglitz GG, Nahai F, Murcia C. Updated international clinical recommendations on scar management: part 1--evaluating the evidence. Dermatol Surg. 2014;40(8):817-824.

6. Gamboa GM, Ross WA. AFT in aesthetic facial recontouring. Ann Plast Surg 2013;70(5):513-516.

7. Shuck J, Iorio ML, Hung R, Davison SP. Autologous fat grafting and injectable dermal fillers for human immunodeficiency virus-associated facial lipodystrophy: a comparison of safety, efficacy, and long-term treatment outcomes. Plast Reconstr Surg. 2013;131(3):499-506. 
8. Klinger M, Caviggiloi F, Klinger FM, et al. Autologous fat graft in scar treatment. $J$ Craniofac Surg. 2013;24(5):1610-1615.

9. Azzam OA, Atta AT, Sobhi RM, Mostafa PI. Fractional CO(2) laser treatment vs autologous fat transfer in the treatment of acne scars: a comparative study. J Drugs Dermatol. 2013;12(1):e7-e13.

10. Sultan SM, Barr JS, Butala P, et al. Fat grafting accelerates revascularization and decreases fibrosis following thermal injury. J Plast Reconstr Aesthet Surg. 2012;65(2):219-227

11. Pallua N, Baroncini A, Alharbi Z, Stromps JP. Improvement of facial scar appearance and microcirculation by autologous lipofilling. J Plast Reconstr Aesthet Surg. 2014;67(8):1033-1037.

12. Guibert M, Franchi G, Ansari E, et al. Fat graft transfer in children's facial malformations: a prospective three-dimensional evaluation. J Plast Reconstr Aesthet Surg. $2013 ; 66(6): 799-804$

13. Liotta DR, Costantino PD, Hiltzik DH. Revising large scars. Facial Plast Surg. 2012;28(5):492-496
14. Lee DW, Kim YS, Park BY. Simultaneous correction of scars and tissue deficiency with composite grafts in secondary cleft lip deformity. J Oral Maxillofac Surg. 2012;70(7):e419-e427.

15. Balkin DM, Samra S, Steinbacher DM. Immediate fat grafting in primary cleft lip repair. J Plast Reconstr Aesthet Surg. 2014;67(12)"1644-1650.

16. Anderson RR, Donelan MB, Hivnor C, et al. Laser treatment of traumatic scars with an emphasis on ablative fractional laser resurfacing: consensus report. JAMA Dermatol. 2014;150(2):187-193.

17. Krakowski AC, Goldenberg A, Eichenfield LF, Murray JP, Shumaker PR. Ablative fractional laser resurfacing helps treat restrictive pediatric scar contractures. Pediatrics. 2014;134(6):e1700-e1705.

18. Cervelli V, Nicoli F, Spallone D, et al. Treatment of traumatic scars using fat grafts mixed with platelet-rich plasma, and resurfacing of skin with the $1540 \mathrm{~nm}$ nonablative laser. Clin Exp Dermatol. 2012;37(1):55-61.

19. Nita AC, Orzan OA, Filipescu M, Jianu D. Fat graft, laser $\mathrm{CO}_{2}$ and platelet-richplasma synergy in scars treatment. J Med Life. 2013;6(4):430-433. 